\title{
Análisis de la satisfacción laboral del profesorado de formación profesional. Análisis factorial confirmatorio
}

\author{
Analysis of job satisfaction among vocational education \\ teachers. Confirmatory factor analysis
}

María González Álvarez*

\section{RESUMEN}

Con objeto de analizar la satisfacción laboral del profesorado que imparte enseñanzas de formación profesional en el sistema educativo de España, se ha realizado un estudio exploratorio de corte transversal, no experimental. Se estudia dicha satisfacción en el ejercicio en cargos de responsabilidad, interrelaciones, instalaciones y recursos, formación, aspectos laborales, valoración del trabajo y situaciones de "acoso, maltrato o agresión" sufridas. Un protocolo elaborado $a d$ hoc fue enviado por correo electrónico a una muestra de centros y cumplimentado por 416 docentes. Se realizaron análisis descriptivos, diferencia de medias y análisis multivariado. Los índices de ajuste del análisis factorial confirmatorio son: $\mathrm{X} 2 / \mathrm{gl}=1.622$, GFI $=.902$, $\mathrm{RMSEA}=.046, \mathrm{CFI}=.976$, $\mathrm{TLI}=.972, \mathrm{IFI}=.976, \mathrm{NFI}=.940, \mathrm{RFI}=.929, \mathrm{PNFI}=.799 . \mathrm{El}$ coeficiente de fiabilidad de Cronbach es .949. El modelo cuenta con buena consistencia interna e índices de bondad de ajuste adecuados. El profesorado de formación profesional tiene una satisfacción "media-alta" con el trabajo que realiza, relaciones con la comunidad educativa, ambiente de trabajo, recursos didácticos y materiales, espacios, instalaciones y horarios. Se valoran como "media" su satisfacción en las relaciones con instituciones, formación inicial, retribuciones y valoración por parte de la comunidad educativa. Siendo "media-baja" la satisfacción sobre aspectos de formación y valoración por parte de las instituciones y posibilidades de promoción. Sufre situaciones de "acoso, mal-
Palabras clave:

satisfacción

laboral, profesorado, análisis factorial confirmatorio, formación profesional.

* Doctora en Filosofía y Ciencias de la Educación. Licenciada en Derecho. Profesora IES “La Quintan” (Langreo-Asturias) España. Contacto: maria.gz.az@gmail.com. ORCID: https://orcid.org/0000-0001-6830-579X 
trato o agresión". Debe mejorarse su satisfacción en las relaciones con las instituciones y la formación, así como eliminar las situaciones de maltrato sufridas. El estudio puede contribuir a mejorar la situación personal y profesional del profesorado y la calidad de la enseñanza.

\section{SUMMARY}

In order to analyze the job satisfaction of teachers who teach vocational training in Spain's educational system, we implemented an exploratory, cross-sectional, non-experimental study. We studied job satisfaction in positions of responsibility, interrelationships, facilities and resources, training, work aspects, job evaluation, and situations of "harassment, mistreatment or aggression" suffered. An ad hoc protocol was sent by e-mail to a sample of centers and completed by 416 teachers. We performed descriptive analysis, mean difference, and multivariate analysis. The fit indices of the confirmatory factor analysis are $\mathrm{X} 2 / \mathrm{gl}=1.622$, GFI $=.902$, RMSEA $=.046, \mathrm{CFI}=.976, \mathrm{TLI}=.972, \mathrm{IFI}=.976, \mathrm{NFI}=.940$, $\mathrm{RFI}=.929$, PNFI $=.799$. The Cronbach's reliability coefficient is .949. The model has good internal consistency and adequate goodness-of-fit indices. Vocational training teachers have a "medium-high" satisfaction with their work, educational community relations, work environment, teaching resources and materials, spaces, facilities, and schedules. A rating of "average" resulted in satisfaction concerning relations with institutions, initial training, remuneration, and educational community evaluation. Satisfaction with aspects of training and evaluation by institutions and promotion possibilities is "medium-low." They suffer situations of "harassment, mistreatment or aggression." Their satisfaction in relations with institutions and training should be improved and eliminate the situations of mistreatment suffered. The study can contribute to improving the personal and professional situation of teachers and the quality of teaching.
Key words: job satisfaction, teachers, confirmatory factor analysis, professional training. 


\section{Introducción}

La satisfacción laboral del profesorado se presenta en la investigación educativa como un tema de gran interés (Cantón-Mayo \& Téllez-Martínez, 2016; Güell, 2014), relacionándose en diversos trabajos con la calidad de la enseñanza (Anaya-Nieto \& López-Martín, 2014; Banerjee, Stearns, Moller \& Mickelson, 2017; Demirtaú, 2010; Gil-Flores, 2017; Persevica, 2011).

Respecto de la definición del concepto "satisfacción laboral del profesorado", no existe unanimidad. Cantón-Mayo y Téllez-Martínez indican que "se percibe como una actitud, una emoción, un sentimiento y relacionada con el clima, con la salud mental y el equilibrio personal" (2016, p. 215). Anaya-Nieto y López-Martín la consideran como "un estado emocional derivado de la evaluación del propio trabajo; esto es, se refiere a cómo la gente se siente en relación con su actividad laboral o con los diferentes aspectos de su trabajo" (2014, p. 436). Toropova, Myrberg \& Johansson (2020) refieren la aportación al concepto realizada por Evans (1997) en su trabajo “Addressing problems of conceptualization and construct validity in researching teachers' job satisfaction", como "a state of mind determined by the extent to which the individual perceives her/his job-related needs to be met". En relación con el constructo sobre la satisfacción laboral señala Güell que "es pluridimensional siendo, por tanto, varias las dimensiones que contribuyen a conseguirla" (2015, p. 22).

Este tema se analiza desde diferentes ópticas y procedimientos en el campo educativo. Así, Sahito y Vaisanen (2019) realizan una revisión de setenta estudios sobre la satisfacción laboral de los docentes en países en vías de desarrollo, señalando su relación con los incentivos, el apoyo, las condiciones laborales, los logros y realización personal y las relaciones. Sims (2018), en su análisis de 35 países del Talis 2013, entiende que la disciplina del alumnado y la cooperación del profesorado se relacionan positivamente con su satisfacción laboral.

En cuanto a los modelos teóricos propuestos para explicar la satisfacción laboral, Anaya-Nieto y López-Martín (2014) entienden que prestan especial atención a distintos factores, tales como las características del trabajo, la autoeficiencia, características personales, expectativas, metas perseguidas o al ajuste persona-ambiente. 
En la literatura, las valoraciones que se realizan sobre la satisfacción laboral del profesorado son diversas. Así en algunos estudios se considera "alta" (Demirtaú, 2010; Naeem-Akhtara, AmirHashmib, \& Hussain-Naqvic, 2010; Persevica, 2011; Schwartz, 2017); en otros se valora como "media-alta" (Anaya-Nieto \& Suárez, 2007; Larkin, Brantley-Dias \& Lokey-Vega, 2016); es "media" para MuñozMenéndez, Gómez-Mármol y Sánchez-Alcaraz (2017); en el trabajo de Cantón-Mayo y Téllez-Martínez (2016) la satisfacción laboral es "media-baja". En este sentido, en el informe Pisa (2018) se indica que, de los 38 países participantes en el Talis 2018, España se encuentra entre los seis con profesorado más "altamente" satisfecho en su función docente. En relación con la evolución de la satisfacción laboral, en determinados estudios longitudinales se constata que ha disminuido en los últimos años (Anaya-Nieto \& López-Martín, 2014; Gil-Flores, 2017; Klassen \& Anderson, 2009; MetLife Foundation, 2012).

El objeto de las investigaciones realizadas es el profesorado que imparte su docencia en educación infantil, primaria y secundaria, mientras que el de formación profesional no se analiza habitualmente. Por esta razón, se entiende que puede ser de interés prestar especial atención a su satisfacción laboral, teniendo en cuenta aportaciones realizadas por los distintos modelos señalados y la experiencia profesional propia.

El estudio tiene como objetivo analizar distintos aspectos que pudieran considerarse integrantes de la satisfacción laboral del profesorado que imparte enseñanzas de formación profesional en el sistema educativo de España. Satisfacción referida a los cargos de responsabilidad en el centro; sus relaciones con las instituciones (administración e inspección de educación) y los miembros de la comunidad educativa (directivos, profesorado, alumnado y familias); formación inicial y permanente recibida; a las instalaciones y recursos del centro para llevar a cabo sus tareas docentes; horario laboral; la participación en los órganos de gobierno del centro y la ubicación del centro. Se analiza, asimismo, la satisfacción del profesorado respecto del reconocimiento que, de su tarea, recibe por parte las instituciones y miembros de la comunidad educativa. Se presta atención a la satisfacción acerca de las posibilidades de promoción profesional y a la evolución de, en 
general, la satisfacción laboral del profesorado indicada en el tiempo. Otro importante tema que se estudia es el de las posibles situaciones de "acoso, maltrato o agresión" sufridas por el profesorado por parte de las instituciones y comunidad educativa.

Las variables sociodemográficas a las que prestamos atención en el estudio se refieren al sexo, tiempo de experiencia docente y tamaño del centro en el que ejerce sus funciones.

Respecto de la satisfacción en función del sexo, no existe acuerdo en la literatura. En algunos estudios las profesoras manifiestan una mayor satisfacción laboral que los profesores (Anaya-Nieto \& LópezMartín, 2014; Gil-Flores, 2017; Martín-Ramos, 2015; Raso-Sánchez, Sola-Martínez e Hinojo-Lucena, 2017; Shobha \& Sarvamangala, 2020; Xin \& MacMillan, 1999); en otros no se encuentran diferencias (Crossman \& Harris, 2006; Muñoz-Menéndez et al., 2017; Skaalvik \& Skaalvik, 2009). Los distintos estudios deben analizarse teniendo en cuenta, en cada caso, la situación de la mujer en general, y de las profesoras en particular, en los países de referencia y en las distintas culturas.

En cuanto al tiempo de experiencia docente, algunos autores constatan que, al aumentar la experiencia docente, disminuye la satisfacción laboral (Anaya-Nieto \& López-Martín, 2014; Anaya-Nieto \& Suárez, 2006; Gil-Flores, 2017; Skaalvic \& Skaalvic, 2009; Xin \& MacMillan, 1999), mientras que otros no encuentran diferencias (Crossman \& Harris, 2006; Demirtaú, 2010).

Teniendo en cuenta el tamaño del centro en el que ejerce sus funciones el profesorado consultado, tampoco se encuentra unanimidad. Según Gil-Flores (2017) y Skaalvic \& Skaalvic (2009), tiene un mayor nivel de satisfacción el profesorado de centros de pequeño tamaño. Martínez-Garrido (2017) considera que se encuentran más satisfechos los docentes que trabajan en escuelas de ciudades grandes frente a los pueblos pequeños.

La satisfacción en relación con el ejercicio en cargos de responsabilidad en el centro es otro aspecto de interés. Dichos cargos se entienden referidos a los directivos (dirección, jefatura de estudios y secretaría), función tutorial, otros cargos (jefatura de departamento) y consejo escolar. En distintas investigaciones aparecen resultados 
diferentes; así, para Papanastasiou y Zembylas (2005) el profesorado que ocupa puestos más altos en las instituciones de educación tiende a estar más satisfecho con su trabajo, mientras que para Ramery-Gelpi (2017) no hay diferencias significativas.

El profesorado desarrolla su tarea profesional en un entorno con amplias relaciones interpersonales. Algunas de estas se refieren a las instituciones (administración educativa e inspección de educación) y a la comunidad educativa (equipo directivo, profesorado del centro, profesorado del departamento y alumnado al que se imparte docencia). Hernández-Silva, Pavez-Lizarraga, González-Donoso y TecpanFlores (2017), y Torres (2010), entienden que el profesorado suele sentirse poco satisfecho en sus relaciones con la administración educativa, considerando al respecto Hernando-Mora y Sanz-Ponce (2015) que la administración debe proporcionar a los docentes más apoyos y recursos. Mientras que, por el contrario, profesores y profesoras se sienten más satisfechos en sus relaciones con el alumnado (Hernández-Silva et al., 2017; Torres, 2010; Veldman, Van Tartwijk, Brekelmas \& Wubbles, 2013).

Los recursos de que dispone el profesorado en su centro para llevar a cabo sus tareas docentes cabe considerarlos como factores que pueden influir en su satisfacción laboral. Se hace referencia en el trabajo a las instalaciones del centro, los recursos materiales y didácticos a disposición del profesorado en el centro, y a los espacios disponibles para desarrollar el trabajo. La organización del centro, la información que se recibe respecto de su funcionamiento y el ambiente de trabajo son otros elementos de interés en el constructo que se plantea sobre la satisfacción laboral del profesorado de formación profesional.

La formación del profesorado es considerada como un elemento fundamental en la calidad de la enseñanza. Así, Ros-Garrido y GarcíaRubio (2016) entienden que, para garantizar la calidad educativa, se debe proporcionar una adecuada formación inicial y permanente al profesorado. Diversos estudios estiman que el profesorado que participa en programas de formación continua se siente más satisfecho (Hernández-Silva et al., 2017; Martínez-Garrido, 2017). En el presente estudio se analiza la satisfacción en la formación en relación con la inicial, permanente en general, función tutorial, normativa educativa, 
materias que se imparten, trabajo en equipo, aspectos de pedagogía y psicología, y gestión de conflictos del alumnado.

Aspectos laborales que pueden considerarse de interés en el análisis de la satisfacción del profesorado son la ubicación del centro y su distancia desde el domicilio, el horario laboral, el número de horas lectivas y su distribución, el horario de reuniones y su número, y las posibilidades de participación en los órganos de gobierno. En algunos estudios las condiciones laborales se relacionan con el estrés y la ansiedad (Collie, Shapka \& Perry, 2012; Ferguson, Frost, \& Hall, 2012), los cuales disminuyen la satisfacción laboral (Klassen, \& Chiu 2010; Liu \& Ramsey, 2008). En diferentes estudios se relaciona la satisfacción laboral con la valoración que se realiza del centro (National Center For Education Statistics, 1997; Santos-Rego, Godás-Otero, Lorenzo-Moledo \& Gómez-Fraguela, 2010). Las condiciones de vida se presentan asociadas, en el caso del profesorado más veterano, a la facilidad de desplazamiento desde el domicilio al centro (Anaya-Nieto \& López-Martín, 2014).

Respecto del importante tema de las retribuciones, en distintos trabajos se encuentra un efecto positivo sobre la satisfacción laboral (Franco-López, López-Arellano y Arango-Botero, 2020; Persevica, 2011), mientras que para Hanushek, Kain y Rivkin (2004) no se encuentra dicha relación.

La satisfacción en relación con reconocimiento y valoración que el profesorado manifiesta tener por parte de las instituciones educativas y de la comunidad educativa, son otros factores analizados en el estudio como componentes del constructo de satisfacción laboral.

Asimismo, se analiza la posibilidad de que el profesorado consultado se sienta acosado, maltratado o agredido por las instituciones (administración educativa e inspección de educación) y por miembros de la comunidad educativa (directivos, profesorado y alumnado). Con el objeto de analizar dicha satisfacción laboral, se lleva a cabo un análisis factorial confirmatorio que puede darnos el constructo que se propone en las cuestiones planteadas en el trabajo.

Se considera que el análisis de la satisfacción laboral del profesorado de enseñanza profesional puede contribuir a mejorar su situación profesional y de la calidad de la enseñanza. Se espera, asimismo, que el trabajo aporte información a las administraciones y comunidad edu- 
cativa para implementar programas que hagan posible la mejora de dicha situación y calidad.

\section{Metodología}

\subsection{Participantes}

Mediante un proceso exploratorio de corte transversal, no experimental, se realiza una consulta al profesorado de Formación Profesional (en adelante FP) del sistema educativo de España, con el objeto de conocer su satisfacción en relación con diversos aspectos de su actividad laboral. Para ello, se elaboró un protocolo ad hoc, junto con una carta que señala los objetivos del trabajo y la importancia de la colaboración solicitada. Sendos documentos se enviaron por correo electrónico a una muestra de centros seleccionados al azar, en función de la población de las distintas provincias. La muestra se obtuvo del Registro Estatal de Centros Docentes no Universitarios del Ministerio de Educación. En este sentido, se tuvieron en consideración 4.323 centros que imparten las enseñanzas señaladas en todo el territorio nacional, enviándose el mensaje al $25 \%$ de los mismos. Las direcciones de correo a las que se realizó el envío corresponden a las oficiales de los centros educativos, custodiadas por la dirección de ellos, siendo ésta la responsable de hacer llegar la consulta al profesorado. Las respuestas se recogieron en una aplicación de Google que nos indicó que el mensaje fue abierto por el $30,3 \%$ de los receptores (327 centros) $)^{2}$. El cuestionario fue cumplimentado por 416 docentes, siendo el $55,1 \%$ profesoras, el $43,9 \%$ profesores, mientras que el $1 \%$ no respondió al sexo. Su edad media es de 47.0 años, desviación típica (en adelante DT) 7.9 años, (profesoras media 46.2 años, DT=7.6, y profesores media 47.9 años, DT=8.1, grado de significación .029, en adelante p). La experiencia profesional media es de 15.1 años, DT=10.2 años (profesoras media 13.7 años, DT=9.8, y profesores media 16.9 años, $\mathrm{DT}=10.5, \mathrm{p}=.002)$. La media del número de alumnos y alumnas de los centros es de 530.6, DT=501.5.

2 Este procedimiento permite dirigir la consulta a un importante número de sujetos, así como garantizar su total anonimato. No obstante, el número de respuestas obtenidas puede verse reducido debido a la desconfianza que en un elevado porcentaje de casos genera el recibir un correo del que se desconoce su origen, eliminándose sin proceder a su apertura y lectura. 


\subsection{Instrumento}

El cuestionario propuesto consta de los siguientes elementos: 1. Variables de clasificación: sexo, tiempo de experiencia docente y número de alumnos y alumnas del centro. 2. Satisfacción en el ejercicio en cargos de responsabilidad (directivos, tutoría, jefatura de departamento y consejo escolar). 3. Satisfacción en las relaciones con la administración e inspección educativa, directivos del centro, profesorado del centro y del departamento y alumnado. 4. Satisfacción con el centro para el desarrollo de las tareas docentes respecto de las instalaciones, recursos didácticos y materiales, organización general del centro, información sobre el funcionamiento del centro, ambiente de trabajo y espacios disponibles para desarrollar el trabajo. 5. Satisfacción con la formación inicial, permanente, función tutorial, normativa educativa, materias que se imparten, trabajo en equipo, aspectos de pedagogía y psicología, y gestión de conflictos del alumnado. 6 . Satisfacción sobre aspectos laborales como la ubicación del centro, retribuciones, horario laboral, número y distribución de horas lectivas, número y distribución de reuniones, posibilidades de participación en los órganos de gobierno, tiempo que se tarda en llegar desde el domicilio al centro, posibilidades de promoción profesional, satisfacción con el trabajo actual que se realiza respecto de la de hace cinco años. 7. Satisfacción en relación con el reconocimiento y valoración que se realiza del trabajo del profesor o profesora por parte de las administraciones educativas, inspección educativa, equipo directivo, profesorado y alumnado. 8. Posibles situaciones de acoso, maltrato o agresión y su grado, sufridas por parte de las administraciones educativas, servicio de inspección educativa, equipos directivos, profesorado y alumnado.

La validez inicial del contenido del cuestionario se analizó mediante "el juicio de expertos": dos inspectores de educación, tres directores y directoras, y cinco profesores y profesoras (que imparten docencia de FP), los cuales valoraron los ítems del cuestionario inicial en una escala de 0 a 10 puntos, siendo 0 el menor valor y 10 el máximo. Se eliminaron los ítems con valoración media inferior a siete puntos. El nuevo cuestionario volvió a ser analizado por los "expertos", prestando atención a la calidad de la redacción de los ítems y aspectos no incluidos en los análisis estadísticos. Con las últimas 
aportaciones se elaboró el cuestionario definitivo, conformado por 36 ítems junto con las variables sociodemográficas, la satisfacción en el ejercicio de cargos de responsabilidad y posibles situaciones de acoso, maltrato o agresión sufridas por el profesorado por parte de las instituciones y comunidad educativa.

Se realizó un análisis factorial exploratorio con los 36 ítems que componían el cuestionario inicial y se eliminaron once, cuyas cargas factoriales eran menores de .60, quedando un total de 25 ítems.

Los eliminados se refieren a la satisfacción que el profesorado tiene respecto de "las relaciones con la administración educativa", "las relaciones con el servicio de inspección", "las relaciones con el alumnado al que se imparte docencia", "la formación inicial respecto a su tarea docente en sus estudios universitarios", "la ubicación del centro en el que se imparte docencia", "las retribuciones como profesor o profesora", "las posibilidades de participación en los órganos de gobierno", "el tiempo que se tarda en llegar desde el domicilio al centro", "el reconocimiento y valoración que el equipo directivo del actual centro realiza del trabajo del docente", "el reconocimiento y valoración que el profesorado del centro realiza del trabajo del docente", y "el reconocimiento y valoración que el alumnado al que se imparte docencia realiza del trabajo del docente".

En relación con la validez del constructo de dichos ítems, la medida de adecuación muestral de Kaiser-Meyer-Olkin (KMO) tiene un valor de .930 y la prueba de esfericidad de Bartlett un grado de significación menor de .001. El método de extracción utilizado ha sido el Análisis de Componentes Principales y el método de rotación Varimax con Kaiser. La rotación converge en siete iteraciones. Cinco factores explican el 70,724\% de la varianza; dichos factores y su varianza son: Factor 1, "Satisfacción con la formación" el 19,951\%. Factor 2, "Satisfacción en las relaciones con las instituciones educativas y directivos del centro" el 17,490\%. Factor 3, "Satisfacción con horarios laborales y reuniones" el 13,324\%, Factor 4, "Satisfacción con las instalaciones y recursos en el centro" el 10,568\% y Factor 5, "Satisfacción con el reconocimiento del trabajo del profesorado por parte de la administración e inspección y posibilidades de promoción" el 9.392\%. 
Tabla 1

Matriz de componentes rotados

\begin{tabular}{|c|c|c|c|c|c|}
\hline Satisfacción laboral con: & 1 & 2 & 3 & 4 & 5 \\
\hline 21.Formación. Pedagogía-psicología & .861 & & & & \\
\hline 20.Formación. Materias que se imparten & .850 & & & & \\
\hline 19.Formación. Conflictos & .837 & & & & \\
\hline 22.Formación. Tutoría & .827 & & & & \\
\hline 17.Formación. Equipo & .812 & & & & \\
\hline 18.Formación. Permanente & .785 & & & & \\
\hline 16.Formación. Normativa & .759 & & & & \\
\hline 27.Horario. Distribución horario lectivo & & .821 & & & \\
\hline 25.Horario. Horario Laboral & & .798 & & & \\
\hline $\begin{array}{l}\text { 28.Horario. Distribución horario de } \\
\text { reuniones }\end{array}$ & & .790 & .336 & & \\
\hline 29.Horario. Número reuniones & & .778 & & & \\
\hline 26.Horario. Número horas lectivas & & .723 & & & \\
\hline 13.Centro. Ambiente trabajo & & & .825 & & \\
\hline $\begin{array}{l}\text { 5. Centro. Relaciones con profesorado } \\
\text { departamento }\end{array}$ & & & .783 & & \\
\hline $\begin{array}{l}\text { 4. Centro. Relaciones con profesorado } \\
\text { centro }\end{array}$ & & & .780 & & \\
\hline $\begin{array}{l}\text { 3. Centro. Relaciones con directivos del } \\
\text { centro }\end{array}$ & & .432 & .674 & & \\
\hline 11.Centro. Organización general centro & & .400 & .642 & .447 & \\
\hline $\begin{array}{l}\text { 12. Centro. Información funcionamiento } \\
\text { centro }\end{array}$ & & .417 & .625 & .303 & \\
\hline 8. Recursos. Instalaciones del centro & & & & .854 & \\
\hline 10.Recursos. Materiales del centro & & & & .835 & \\
\hline 9. Recursos. Didácticos del centro & & & & .826 & \\
\hline $\begin{array}{l}\text { 14. Recursos. Espacios para desarrollar el } \\
\text { trabajo }\end{array}$ & & & & .667 & \\
\hline $\begin{array}{l}\text { 33. Reconocimiento. Por parte de la } \\
\text { inspección }\end{array}$ & .372 & & & & .758 \\
\hline $\begin{array}{l}\text { 32. Reconocimiento. Por parte de la } \\
\text { administración }\end{array}$ & .404 & & & & .737 \\
\hline 38.Promoción profesional & .386 & & & & .653 \\
\hline Alpha de Cronbach .949 & .943 & .907 & .904 & .909 & .881 \\
\hline
\end{tabular}


Para calcular la fiabilidad de la prueba se utiliza el coeficiente de Cronbach, el cual alcanza un valor de Alpha=.949 (en la escala de 0 a 1) para el total de los elementos analizados.

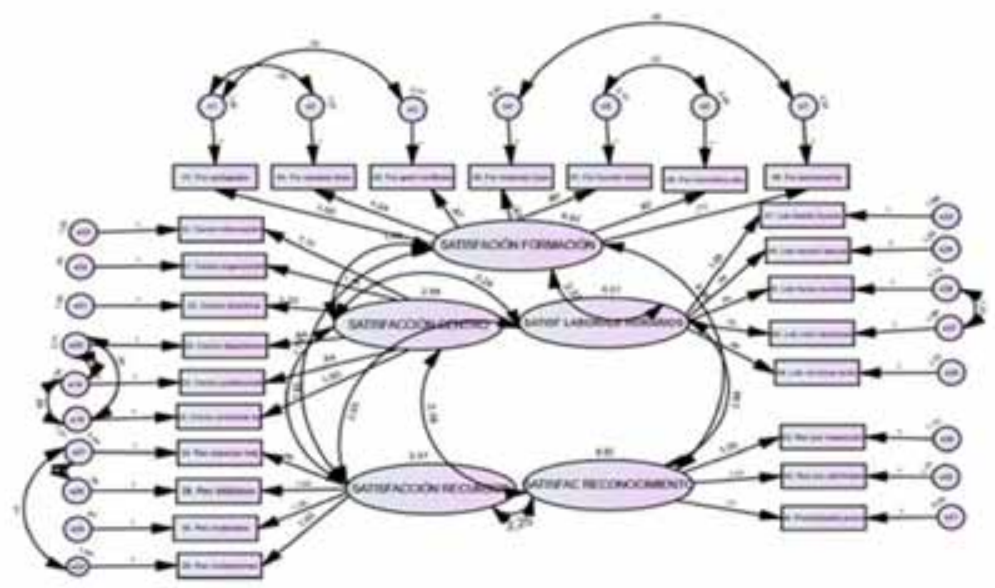

Figura 1. Gráfico análisis factorial confirmatorio Fuente: SPSS AMOS 26.

Se realiza el análisis factorial confirmatorio mediante SPSS AMOS 26 con 300 sujetos, utilizando el método de máxima verosimilitud (ML). Los índices de ajuste obtenidos son: índices de ajuste absoluto chi cuadrado sobre grados libertad X2/gl=1.622; índice de bondad de ajuste GFI=.902, error cuadrático medio de aproximación RMSEA=.046; índice de ajuste comparativo $\mathrm{CFI}=.976$; índice de TukerLewis TLI=.972; índice IFI=.976; índice NFI=.940; índice RFI=.929; índice normado de parsimonia PNFI=.799. Se considera que el modelo cuenta con buena consistencia interna e índices de bondad de ajuste adecuados.

\subsection{Método de análisis}

La información resultante del cuestionario se analiza mediante descriptivos, diferencia de medias (utilizando la prueba $\mathrm{T}$ de muestras independientes) y análisis multivariado (ANOVA de una vía con datos independientes, utilizando el método post-hoc Scheffe y análisis factorial exploratorio y confirmatorio). Se propone para las respuestas, como se ha indicado, una escala de 0 a 10 puntos, siendo 0 el 
menor valor y 10 el máximo. Según la experiencia docente, se forman tres grupos de iguales proporciones, hasta 12 años, de 13 a 23 años y de 24 o más años. En relación con el número de alumnos y alumnas del centro, el primer grupo se establece hasta 249 , el segundo de 250 a 599 y el tercero de 600 o más. Respecto de la evolución de la satisfacción laboral en los últimos cinco años, se proponen cinco opciones para su valoración: 1: "es mucho mejor"; 2: "es bastante mejor"; 3 : "es igual"; 4: "es bastante peor"; 5: "es mucho peor". En relación con "las posibles situaciones de acoso, maltrato o agresión sufridas por el profesorado", se establecen para las respuestas las opciones "sí" o "no". Para las afirmativas se ofrece una escala de cuatro valores de "muy grave", bastante grave", "algo grave" y "leve", para que se indique la gravedad de las situaciones sufridas.

Cuando se señala que las diferencias analizadas no son significativas se hace referencia al nivel de significación p>.050.

\section{Resultados}

\subsection{Satisfacción del profesorado de FP en su trabajo}

El profesorado consultado que imparte docencia de FP tiene una satisfacción laboral media de 8.1 puntos respecto del trabajo que realiza en la escala señalada (DT=1.5); solo el $2,2 \%$ otorga una puntuación menor de 5 puntos. No se encuentran diferencias ( $p>050$ ) según el sexo, grupos de experiencia docente y tamaño del centro.

\subsection{Satisfacción del profesorado de FP según experiencia en el ejercicio de cargos directivos, función tutorial, otros cargos y en el consejo escolar}

Se considera de interés, en el análisis de la satisfacción laboral del profesorado, conocer ésta según la experiencia en cargos de responsabilidad en el centro, tales como dirección, función tutorial, otros cargos y el consejo escolar del centro.

El 19,8\% del profesorado de FP manifiesta tener experiencia en cargos directivos (dirección, jefatura de estudios o secretaría), con un tiempo medio de 6.8 cursos ( $\mathrm{DT}=6.3$ ), valorando su satisfacción en ellos con 6.8 puntos de media (DT=2.1). 
Tienen experiencia en la función tutorial el 91.8\%, durante un tiempo medio de 8.5 cursos (DT=7.9) y una satisfacción de 7.2 puntos de media (DT=1.8).

En otros cargos (jefatura de departamento, etc.), señala tener experiencia el $53 \%$ del profesorado, siendo su duración media de 5.3 cursos, $\mathrm{DT}=4.7$ y con una satisfacción media de 7.0 puntos, $\mathrm{DT}=2.0$.

El 28\% del profesorado indica haber pertenecido o pertenecer al consejo escolar del centro, durante una media de 6.1 cursos (DT=6.5), valorando su satisfacción en él con 5.7 puntos de media (DT=2.7).

No se encuentran diferencias ( $\mathrm{p}>$.050) respecto de la satisfacción en dichos cargos en función de las variables analizadas de sexo, grupos de experiencia docente y tamaño del centro.

Tabla 2

Experiencia y satisfacción del profesorado de FP en el ejercicio de cargos de responsabilidad en los centros

\begin{tabular}{lllllll}
\hline $\begin{array}{l}\text { Experiencia y } \\
\text { satisfacción del } \\
\text { profesorado de FP en: }\end{array}$ & $\begin{array}{l}\text { Experiencia } \\
\text { Sí }\end{array}$ & $\begin{array}{l}\text { Número de } \\
\text { cursos }\end{array}$ & Satisfacción & \\
\cline { 2 - 7 } & $\%$ & Media & DT & Media & DT & $\begin{array}{l}\text { Menos } \\
\text { de 5 } \\
\text { puntos }\end{array}$ \\
\hline 1. Cargos directivos & 19,8 & 6.8 & 6.3 & 6.8 & 2.1 & 12.5 \\
\hline 2. Función tutorial & 91,8 & 8.5 & 7.9 & 7.2 & 1.8 & 7.6 \\
\hline 3. Otros cargos & 53 & 5.3 & 4.7 & 7.0 & 2.0 & 9.4 \\
\hline 4. Consejo escolar & 28, & 6.1 & 6.5 & 5.7 & 2.7 & 24.6 \\
\hline
\end{tabular}

\subsection{Satisfacción del profesorado de FP en sus relaciones interpersonales}

En el ámbito laboral, y en concreto en el del profesorado, se considera de gran importancia la satisfacción que se encuentra en las relaciones interpersonales. En este caso se tienen en cuenta las referidas a la administración educativa, inspección educativa, directivos del centro, profesorado del centro en general y del departamento en particular, y del alumnado al que se imparte docencia.

La satisfacción mejor valorada en las relaciones interpersonales del profesorado de FP se refiere al "profesorado del centro en general" (7.8 media, DT=1.5) y al "profesorado del departamento" (7.8 media, 
DT=1.9). La satisfacción con "los directivos del centro" se valora con 7.3 de media (DT=2.5) y "el alumnado al que se imparte docencia" obtiene igual media (DT=1.8). Las relaciones con "la administración educativa" se valora con media de 6.1 (DT=2.3) y la satisfacción con "la inspección educativa" con 5.5 puntos (DT=2.6). Las relaciones con "el profesorado" son mejor valoradas por las profesoras (7.9 media, DT=1.5) que por los profesores (7.6 media, DT=1.5), $\mathrm{p}=.019$. Las profesoras (7.6 media, DT=1.7) también se manifiestan más satisfechas en sus relaciones con "el alumnado" que los profesores (7.0 media, DT=1.9), p=.002.

No se encuentran diferencias ( $p>$.050) en la satisfacción del profesorado de FP en sus relaciones con la administración educativa, directivos del centro y profesorado del departamento, según las variables sociodemográficas propuestas.

Tabla 3

Satisfacción del profesorado de FP en sus relaciones interpersonales

\begin{tabular}{llll}
\hline $\begin{array}{l}\text { Satisfacción del profesorado de FP en sus } \\
\text { relaciones con: }\end{array}$ & Media & DT & $\begin{array}{l}\text { Menos de 5 } \\
\text { puntos } \\
\%\end{array}$ \\
\hline 1. La administración educativa & 6.1 & 2.3 & 20,7 \\
\hline 2. La inspección educativa & 5.5 & 2.6 & 26,9 \\
\hline 3. Los directivos del centro & 7.3 & 2.5 & 14,0 \\
\hline 4. El profesorado del centro & 7.8 & 1.5 & 3,5 \\
\hline 5. El profesorado del departamento & 7.8 & 1.9 & 6,0 \\
\hline 6. El alumnado al que se imparte docencia & 7.3 & 1.8 & 7,7 \\
\hline
\end{tabular}

3.4. Satisfacción del profesorado de FP con la organización, el ambiente de trabajo, instalaciones y recursos del centro

Se considera que la satisfacción del profesorado de FP puede verse relacionada con aspectos laborales del centro, como son las instalaciones en relación con sus tareas docentes, los recursos materiales y didácticos de que se dispone en el centro, la organización general, la información que se recibe sobre el funcionamiento del centro, el ambiente de trabajo y los espacios de que se dispone para desarrollar el trabajo.

La satisfacción con "el ambiente de trabajo" se valora por el profesorado con 7.4 puntos de media (DT=2.0). La satisfacción con "la organización general del centro" y "la información sobre el funciona- 
miento del centro" recibe 7.0 de media (DT=2.0 y DT=2.2, respectivamente). Con "los recursos didácticos del centro" obtiene 6.6 de media (DT=1.9); "los espacios de que se dispone para el desarrollo del trabajo", 6.5 (DT=2.2); "los recursos materiales del centro", 6.4 (DT=2.0) y "las instalaciones del centro", 6.3 puntos de media (DT=2.1).

No se establecen diferencias ( $\mathrm{p}>$.050) en la satisfacción del profesorado con relación a los aspectos estudiados en función del sexo, tiempo de experiencia docente y tamaño del centro.

Tabla 4.

Satisfacción del profesorado con la organización, el ambiente de trabajo, instalaciones y recursos en el centro

\begin{tabular}{llll}
\hline $\begin{array}{l}\text { Satisfacción del profesorado en relación con el } \\
\text { centro respecto de: }\end{array}$ & Media & DT & $\begin{array}{l}\text { Menos de } \\
\text { 5 puntos } \\
\%\end{array}$ \\
\hline 1. Las instalaciones & 6.3 & 2.1 & 19,2 \\
2. Los recursos didácticos & 6.6 & 1.9 & 11,7 \\
3. Los recursos materiales & 6.4 & 2.0 & 16.5 \\
4. La organización general & 7.0 & 2.0 & 11,5 \\
5. La información que se recibe sobre su & 7.0 & 2.2 & 11,7 \\
$\quad$ funcionamiento & & & \\
6. El ambiente de trabajo & 7.4 & 2.0 & 9,5 \\
7. Los espacios de que se dispone para & 6.5 & 2.2 & 16,7 \\
$\quad$ desarrollar el trabajo & & & \\
\hline
\end{tabular}

\subsection{Satisfacción del profesorado de FP con la formación recibida}

Para conocer la satisfacción con la formación, tanto inicial como permanente, del profesorado de FP se analizan diferentes aspectos de ésta, tales como la formación inicial de los estudios universitarios respecto de su tarea docente, la formación permanente en general en relación con el trabajo, la función tutorial, la normativa educativa, las materias que se imparten como profesor o profesora, el trabajo en equipo, aspectos de pedagogía y psicología, y la gestión de conflictos del alumnado.

La satisfacción con "la formación permanente en relación con el trabajo" (6.1 media, DT=2.3) es la mejor valorada de los aspectos formativos analizados del profesorado de FP. "La formación inicial de los estudios universitarios" (5.7 media, DT=2.7) y "la formación en las materias que se 
imparten como profesor o profesora" (5.2 media, DT=2.7) obtienen valoraciones superiores a cinco puntos. Los restantes aspectos analizados tienen menos de cinco puntos: "la función tutorial" (4.7 media, DT=2.5), "la normativa educativa" (4.6 media, DT=2.6), "el trabajo en equipo (4.4 media, DT=2.8), "la gestión de conflictos del alumnado" (4.2 media, DT=2.7) y "aspectos de pedagogía y psicología” (4.1 media, DT=2.7).

No se encuentran diferencias ( $\mathrm{p}>$.050) con la formación recibida por el profesorado de FP en función del sexo, experiencia docente y tamaño del centro

Tabla 5.

Satisfacción del profesorado de FP con la formación recibida

\begin{tabular}{llll}
\hline Satisfacción con la formación recibida respecto de: & Media & DT & $\begin{array}{l}\text { Menos de 5 } \\
\text { puntos \% }\end{array}$ \\
\hline 1. La inicial de los estudios universitarios & 5.7 & 2.5 & 25,2 \\
2. La permanente en relación con el trabajo & 6.1 & 2.3 & 21,2 \\
3. La función tutorial & 4.7 & 2.5 & 37,7 \\
4. La normativa educativa & 4.6 & 2.6 & 43,1 \\
5. Las materias que imparte como profesor o & 5.2 & 2.7 & 33,9 \\
$\quad$ profesora & & & \\
6. El trabajo en equipo & 4.4 & 2.8 & 45,6 \\
7. Aspectos de pedagogía y psicología & 4.1 & 2.7 & 51,1 \\
8. La gestión de conflictos del alumnado & 4.2 & 2.7 & 49,4 \\
\hline
\end{tabular}

\subsection{Satisfacción del profesorado de FP en determinados aspectos laborales}

Importantes aspectos laborales del profesorado que imparte docencia de FP pueden relacionarse con su satisfacción como docente. Se hace referencia a elementos como la propia ubicación del centro y su distancia al domicilio, las retribuciones percibidas, el horario de permanencia en el centro, el número de horas lectivas y su distribución, el número y horario de reuniones y las posibilidades de promoción profesional. Por otro lado, se analiza la satisfacción del profesorado respecto del reconocimiento y valoración que de su trabajo realizan las administraciones educativas, la inspección de educación, el equipo directivo y profesorado del centro y el alumnado al que se imparte docencia. La evolución en el tiempo de dicha satisfacción laboral es otro elemento que se tiene en cuenta. 
"La ubicación del centro" es el aspecto laboral mejor valorado por el profesorado, con 7.4 puntos de media ( $\mathrm{DT}=2.3)$. Le sigue "el horario laboral de permanencia en el centro", con 7.3 de media (DT=2.2). La satisfacción laboral respecto de "la distribución del horario lectivo" (DT=2.4) y "el número de reuniones" ( $\mathrm{DT}=2.1$ ) se valoran con 7.0 puntos de media. "El horario de reuniones" (DT=2.2) y "el tiempo que se tarda en llegar desde el domicilio al centro" (DT=3.0) reciben una valoración de 6.9 de media. "El número de horas lectivas" que tiene el profesorado se valora con 6.8 de media (DT=2.3). La satisfacción respecto de "las posibilidades de participación en los órganos de gobierno es valorada con 6.6 de media. "Las retribuciones" es el aspecto menos valorado, con 6.4 puntos de media (DT=2.2).

Por otro lado, respecto de la satisfacción que manifiesta tener el profesorado en relación con el reconocimiento y valoración que realizan de su trabajo instituciones y comunidad educativa, se observa que la mejor es la recibida por parte del "alumnado al que se imparte docencia" (7.1 media, DT=1.9), seguida del "profesorado del centro" (6.8 media, DT=2.2) y del "equipo directivo del centro" (6.3 media, DT=2.8). "Las administraciones educativas" (4.0 media, DT=2.8) y "la inspección educativa" (4.2 media, DT=2.8) tienen una valoración inferior a cinco puntos de media de satisfacción.

En referencia a "las posibilidades de promoción profesional", la valoración media de su satisfacción es de 4.6 puntos, DT=2.8.

El 42,9\% del profesorado manifiesta que su satisfacción laboral actual, respecto de la de hace cinco años, es "mucho o bastante mejor". Algo más de uno de cada tres $(35,9 \%)$ estima que es "igual" y el 21,2\% entiende que es "bastante o mucho peor".

Con relación a las "retribuciones" que perciben en su tarea profesional, las profesoras se sienten más satisfechas (6.8 media, $\mathrm{DT}=2.1$ ) que los profesores (6.1 media, DT=2.3), $\mathrm{p}=.001$.

La "ubicación del centro" es más satisfactoria para el profesorado de mayor experiencia profesional, 24 o más años, (7.7 media, DT=2.0) que para el de menor experiencia, hasta 12 años, (7.0 media, DT=2.5), $\mathrm{p}=.043$. De igual manera, se establecen diferencias con relación "al tiempo que se tarda en llegar del domicilio al centro", mayor experiencia (7.6 media, DT=2.6) y menor experiencia (6.4 media, $\mathrm{DT}=3.3$ ), $\mathrm{p}=.004$. 
No se encuentran diferencias ( $p>050)$ en las valoraciones de los restantes aspectos estudiados según las variables analizadas del sexo, tiempo de experiencia docente y tamaño del centro.

Tabla 6

Satisfacción sobre aspectos laborales del profesorado de FP

\begin{tabular}{|c|c|c|c|c|}
\hline $\begin{array}{l}\text { Satisfacción sobre aspectos laborales del } \\
\text { profesorado de FP respecto de: }\end{array}$ & Media & DT & \multicolumn{2}{|c|}{$\begin{array}{l}\text { Menos de } 5 \text { puntos } \\
\%\end{array}$} \\
\hline 1. La ubicación del centro & 7.4 & 2.3 & \multicolumn{2}{|l|}{11,2} \\
\hline 2. Las retribuciones & 6.4 & 2.2 & \multicolumn{2}{|l|}{16,7} \\
\hline $\begin{array}{l}\text { 3. El horario laboral de permanencia en } \\
\text { el centro }\end{array}$ & 7.3 & 2.2 & \multicolumn{2}{|l|}{10,7} \\
\hline 4. El número de horas lectivas & 6.8 & 2.3 & \multicolumn{2}{|l|}{15,7} \\
\hline 5. La distribución del horario lectivo & 7.0 & 2.4 & \multicolumn{2}{|l|}{15,5} \\
\hline 6. El horario de reuniones & 6,9 & 2.2 & \multicolumn{2}{|l|}{11,5} \\
\hline 7. El número de reuniones & 7.0 & 2.1 & \multicolumn{2}{|l|}{12} \\
\hline $\begin{array}{l}\text { 8. Posibilidades de participación en los } \\
\text { órganos de gobierno }\end{array}$ & 6.6 & 2.3 & \multicolumn{2}{|l|}{12,5} \\
\hline $\begin{array}{l}\text { 9. Tiempo que se tarda desde el domicilio } \\
\text { al centro }\end{array}$ & 6.9 & 3.0 & \multicolumn{2}{|l|}{20,7} \\
\hline $\begin{array}{l}\text { 10. Posibilidades de promoción } \\
\text { profesional }\end{array}$ & 4.6 & 2.8 & \multicolumn{2}{|l|}{42,1} \\
\hline $\begin{array}{l}\text { 11. Reconocimiento y valoración que se } \\
\text { realiza del trabajo del profesorado por } \\
\text { parte de: }\end{array}$ & Media & DT & \multicolumn{2}{|c|}{$\begin{array}{l}\text { Menos de } 5 \text { puntos } \\
\%\end{array}$} \\
\hline 11. 1. Las administraciones educativas & 4.0 & 2.8 & \multicolumn{2}{|l|}{51,9} \\
\hline 11. 2. La inspección educativa & 4.2 & 2.8 & \multicolumn{2}{|l|}{46,9} \\
\hline 11. 3. El equipo directivo & 6.3 & 2.8 & \multicolumn{2}{|l|}{20} \\
\hline 11.4. El profesorado del centro & 6.8 & 2.2 & \multicolumn{2}{|l|}{12} \\
\hline $\begin{array}{l}\text { 11.5. El alumnado al que imparte } \\
\text { docencia }\end{array}$ & 7.1 & 1.9 & \multicolumn{2}{|l|}{9,7} \\
\hline \multirow{3}{*}{$\begin{array}{l}\text { 12. La satisfacción laboral actual respecto } \\
\text { a la de hace cinco años es: }\end{array}$} & 2.6 & 1.1 & \multicolumn{2}{|l|}{-----} \\
\hline & \multirow[t]{2}{*}{$\%$} & $\%$ & & \\
\hline & & $\begin{array}{l}\text { "Mucho } \\
\text { o } \\
\text { bastante } \\
\text { mejor" }\end{array}$ & Igual & $\begin{array}{l}\text { "Bastante } \\
\text { o mucho } \\
\text { peor" }\end{array}$ \\
\hline 13.1. Mucho mejor & 19,5 & \multirow[t]{5}{*}{42,9} & \multirow[t]{5}{*}{35,9} & \multirow[t]{5}{*}{21,2} \\
\hline 13.2. Bastante mejor & 23,4 & & & \\
\hline 13.3. Semejante & 35,9 & & & \\
\hline 13.4. Bastante peor & 14 & & & \\
\hline 13.5. Mucho peor & 7,2 & & & \\
\hline
\end{tabular}




\subsection{Posibles situaciones de acoso, maltrato o agresión sufridas por el profesorado de FP por parte de las instituciones y comunidad educativa}

Las situaciones de "acoso, maltrato o agresión" por parte de las instituciones (administración educativa e inspección) y comunidad educativa (directivos, profesorado y alumnado) al profesorado de FP, se estudian para conocer su situación y poder, en posteriores trabajos, analizar su relación con su satisfacción laboral.

El profesorado responde si ha sufrido o sufre situaciones de "acoso, maltrato o agresión" por parte de "los responsables de las administraciones educativas", "los miembros del servicio de inspección educativa", "los miembros de los equipos directivos", "los profesores y profesoras", y "los alumnos y alumnas" con las opciones "sí" o "no". Las respuestas afirmativas se valoran en una escala de cuatro valores: "muy grave", bastante grave", "algo grave" y "leve".

Manifiestan sentirse "acosado, maltratado o agredido" por "los alumnos o alumnas" el 26,2\% del profesorado; uno de cada cinco $(20,5 \%)$ por "los miembros de los equipos directivos"; por "los profesores o profesoras", el 16,4\%; por "los responsables de la administración educativa", el 10\%, y por "los miembros del servicio de inspección educativa", el 6,8\%.

Tabla 7

Posibles situaciones de acoso, maltrato o agresión sufridas por el profesorado de FP por parte de las instituciones y comunidad educativa

\begin{tabular}{|c|c|c|c|c|c|}
\hline \multirow{3}{*}{$\begin{array}{l}\text { Posibles situaciones de acoso, } \\
\text { maltrato o agresión sufridas por el } \\
\text { profesorado de FP por parte de: }\end{array}$} & \multicolumn{5}{|l|}{$\begin{array}{l}\text { Sí } \\
\%\end{array}$} \\
\hline & \multirow[t]{2}{*}{$\%$} & \multicolumn{4}{|c|}{ Grado de "acoso, maltrato o agresión" } \\
\hline & & $\begin{array}{l}\text { 1. Muy } \\
\text { grave }\end{array}$ & $\begin{array}{l}2 . \\
\text { Bastante } \\
\text { grave }\end{array}$ & $\begin{array}{l}3 . \\
\text { Algo } \\
\text { grave }\end{array}$ & $\begin{array}{l}4 . \\
\text { Leve }\end{array}$ \\
\hline $\begin{array}{l}\text { 1. Responsables de la administración } \\
\text { educativa }\end{array}$ & 10 & 0,7 & 2,3 & 2,3 & 4,7 \\
\hline $\begin{array}{l}\text { 2. Miembros del servicio de } \\
\text { inspección educativa }\end{array}$ & 6,8 & 0,7 & 2,1 & 1,3 & 2,7 \\
\hline $\begin{array}{l}\text { 3. Miembros de los equipos } \\
\text { directivos }\end{array}$ & 16,8 & 1,2 & 4,5 & 5,4 & 5,7 \\
\hline 4. Profesores y profesoras & 19,5 & 3,4 & 6,2 & 4,6 & 5,3 \\
\hline 5. Alumnos y alumnas & 26,2 & 2,6 & 5,1 & 6,3 & 12,2 \\
\hline
\end{tabular}




\subsubsection{Posibles situaciones de "acoso, maltrato o agresión"} sufridas por el profesorado de FP por parte de las instituciones y comunidad educativa, según sexo

Las profesoras sufren situaciones de "acoso, maltrato o agresión" por parte de "los responsables de la administración educativa" y de "los miembros del servicio de inspección educativa” en menor porcentaje que los profesores. Por el contrario, las profesoras manifiestan sufrir en mayor porcentaje dichas situaciones por parte de "los miembros de los equipos directivos" y de "los alumnos y alumnas". No se manifiestan diferencias entre profesoras y profesores respecto de las situaciones de "acoso, maltrato o agresión" sufridas por parte de "los profesores y profesoras".

Tabla 8

Posibles situaciones de acoso, maltrato o agresión sufridas por el profesorado de FP por parte de las instituciones y comunidad educativa, según sexo

\begin{tabular}{llll}
\hline $\begin{array}{l}\text { Posibles situaciones de "acoso, maltrato o } \\
\text { agresión" sufridas por el profesorado de }\end{array}$ & $\begin{array}{l}\text { Total } \\
\text { FP según sexo por parte de: }\end{array}$ & $\begin{array}{l}\text { Profesoras } \\
\%\end{array}$ & $\begin{array}{l}\text { Profesores } \\
\%\end{array}$ \\
\hline $\begin{array}{l}\text { 1. Responsables de la administración } \\
\text { educativa }\end{array}$ & 10 & 7,3 & 13,1 \\
\hline $\begin{array}{l}\text { 2. Miembros del servicio de inspección } \\
\text { educativa }\end{array}$ & 6,8 & 4,6 & 9,2 \\
\hline 3. Miembros de los equipos directivos & 16,8 & 18,4 & 14,8 \\
\hline $\begin{array}{l}\text { 4. Profesores y profesoras } \\
\text { 5. Alumnos y alumnas }\end{array}$ & 19,5 & 19,7 & 19,3 \\
\hline
\end{tabular}

3.7.2. Posibles situaciones de "acoso, maltrato o agresión" sufridas por el profesorado de FP por parte de las instituciones y comunidad educativa, según tiempo de experiencia docente

Según manifiesta el profesorado consultado, al aumentar el tiempo de experiencia docente se sufre mayor porcentaje de "acoso, maltrato o agresión" por parte de "los responsables de la administración educativa". Así, sufre estas situaciones el 7,1\% de los docentes que tienen hasta 12 años de experiencia, el 11,0\% del profesorado que tiene de 13 a 23 años y el 14,7\% de los que ha ejercido sus funciones durante 24 o más años.

De igual manera, con el aumento de experiencia docente es mayor el porcentaje de profesores y profesoras que manifiestan sentirse "acosados, maltratados o agredidos" por parte de "los miembros del 
servicio de inspección educativa". El porcentaje es del 3,8\% en el caso de los docentes que tienen hasta 12 años de experiencia, del 8,5\% en los que tienen de 13 a 23 años, y del 10,5\% en los que han ejercido sus funciones durante 24 años o más.

El profesorado de menor experiencia manifiesta sufrir estas situaciones por parte de "los miembros de los equipos directivos" en menor porcentaje que los de mayor experiencia. En este sentido se manifiesta el $12 \%$ de los docentes de hasta 12 años, y el $22,0 \%$ y $21,9 \%$, respectivamente, de los restantes grupos.

No se observan importantes diferencias en relación con los porcentajes de profesorado que sufre situaciones de "acoso, maltrato, o agresión" por parte de "los profesores y profesoras".

El profesorado de menor experiencia (hasta 12 años) sufre estas situaciones por parte de "los alumnos y alumnas" en menor porcentaje $(23,9 \%)$ que los dos restantes grupos (28\% de 13 a 23 años y $29,2 \%$ de 24 o más).

Tabla 9

Posibles situaciones de acoso, maltrato o agresión sufridas por el profesorado de FP por parte de las instituciones y comunidad educativa, según tiempo de experiencia docente

\begin{tabular}{lllll}
\hline $\begin{array}{l}\text { Posibles situaciones de "acoso, maltrato o } \\
\text { agresión" sufridas por el profesorado de FP }\end{array}$ & $\begin{array}{l}\text { Total } \\
\text { según tiempo de experiencia docente por } \\
\text { parte de: }\end{array}$ & $\begin{array}{l}\text { Hasta } \\
12 \\
\text { años } \\
\%\end{array}$ & $\begin{array}{l}\text { De } 13 \\
\text { a 23 } \\
\text { años } \\
\%\end{array}$ & $\begin{array}{l}\text { 24 o } \\
\text { más } \\
\text { años } \\
\%\end{array}$ \\
\hline $\begin{array}{l}\text { 1. Responsables de la administración } \\
\text { educativa }\end{array}$ & 10,0 & 7,1 & 11,0 & 14,7 \\
\hline $\begin{array}{l}\text { 2. Miembros del servicio de inspección } \\
\text { educativa }\end{array}$ & 6,8 & 3,8 & 8,5 & 10,5 \\
\hline 3. Miembros de los equipos directivos & 16,8 & 12,0 & 22,0 & 21,3 \\
\hline 4. Profesores y profesoras & 19,5 & 19,1 & 18,8 & 21,9 \\
\hline 5. Alumnos y alumnas & 26,2 & 23,9 & 28,0 & 29,2 \\
\hline
\end{tabular}

\section{Discusión}

La satisfacción del profesorado de FP en su trabajo, analizada en el presente estudio, tiene una valoración que podemos considerar como "alta", coincidiendo con lo señalado en diversos trabajos (Demirtaú, 2010; Naeem-Akhtara et al., 2010; Persevica, 2011; Schwartz, 2017), así 
como en el informe Pisa (2019), y con un valor superior a las investigaciones de Anaya-Nieto y Suárez (2007), Larkin et al. (2016), MuñozMenéndez et al. (2017), Sotomayor-Atahuamán (2020) y Cantón-Mayo y Téllez-Martínez (2016).

Se ha señalado en distintos estudios que la satisfacción laboral del profesorado ha disminuido en los últimos años (Anaya-Nieto \& López-Martín, 2014; Gil-Flores, 2017; Klassen \& Anderson, 2009; MetLife Foundation, 2012), en el caso presente se observa que el porcentaje de profesores y profesoras de FP que entienden que ha mejorado es superior al que considera que ha empeorado.

Respecto de las posibles diferencias según el sexo, profesores y profesoras de FP consultados no presentan diferencias respecto a su satisfacción laboral, coincidiendo con lo propuesto por Crossman y Harris (2006), Muñoz-Menéndez et al. (2017), Skaalvik y Skaalvik (2009), y disintiendo de lo señalado en otras investigaciones, en las que las profesoras manifiestan una mayor satisfacción laboral que los profesores (Anaya-Nieto \&López-Martín, 2014; Gil-Flores, 2017; Gius, 2015; Martín-Ramos, 2015; Naeem-Akhtara et al., 2010; RasoSánchez et al., 2017; Xin \& MacMillan, 1999). Tampoco se encuentran diferencias en el presente estudio en la satisfacción laboral en función del tiempo de experiencia docente, estando de acuerdo con lo indicado por Crossman y Harris (2006), Demirtaú (2010), y a diferencia de otros trabajos para los que, al aumentar la experiencia docente, disminuye la satisfacción laboral (Anaya-Nieto \& López-Martín, 2014; AnayaNieto \& Suárez, 2006; Gil-Flores, 2017; Skaalvic \& Skaalvic, 2009; Xin $\&$ MacMillan, 1999). En el presente trabajo, el tamaño del centro tampoco es una variable respecto de la que se establezcan diferencias; por el contrario, Gil-Flores (2017) y Skaalvic y Skaalvic (2009) entienden que el profesorado de centros de pequeño tamaño tiene un mayor nivel de satisfacción. Debe tenerse en cuenta, en el análisis de dicha satisfacción en función de las variables estudiadas, que los estudios están condicionados por las circunstancias en las que desarrolla su actividad profesional el profesorado consultado, en función del país y de la cultura. El profesorado de FP se manifiesta poco satisfecho en su relación con la administración educativa, otorgando, asimismo, una puntuación "media-baja" a la satisfacción respecto de la valoración y reconocimiento que reciben por parte de dicha administración acer- 
ca de su tarea, al igual que ocurre en los trabajos de Hernández-Silva et al. (2017) y Torres (2010). Dicho profesorado se manifiesta más satisfecho en sus relaciones con el alumnado, estando de acuerdo con lo señalado en distintas investigaciones (Hernández-Silva et al., 2017; Torres, 2010; Veldman et al., 2013).

Por otro lado, se valora como "media-alta" la satisfacción con los recursos existentes en el centro para desarrollar las tareas docentes. Hernando-Mora y Sanz-Ponce (2015) consideran que el profesorado entiende que se le deben de proporcionar más apoyos y recursos.

El profesorado de FP valora su satisfacción con la formación recibida acerca de distintos aspectos formativos como "media-baja". Diversos estudios estiman que la participación del profesorado en programas de formación son un elemento de satisfacción profesional (Hernández-Silva et al., 2017; Martínez-Garrido, 2017).

Es valorada como "media-alta" la satisfacción respecto de aspectos laborales, como el horario, reuniones y posibilidades de participación en el centro, los cuales se relacionan en distintos trabajos con la satisfacción laboral (National Center For Education Statistics, 1997; SantosRego et al., 2010).

El profesorado más veterano está más satisfecho con la ubicación de su centro y con la distancia de éste a su domicilio, así se entiende por Anaya-Nieto y López-Martín, 2014. Es evidente que el profesorado se va aproximando, a lo largo de su carrera profesional, a centros más cercanos a su domicilio o a un domicilio próximo al centro.

Se encuentra un importante número de casos de profesores y profesoras que manifiestan sentirse "acosados, maltratados o agredidos" por instituciones educativas o miembros de la comunidad educativa. En algunos estudios, las condiciones laborales se relacionan con el estrés y la ansiedad, los cuales disminuyen la satisfacción laboral (Klassen, \& Chiu, 2010; Liu \& Ramsey, 2008).

\section{Conclusiones}

El profesorado de FP tiene una satisfacción "media-alta" respecto del trabajo que realiza, no encontrándose diferencias según el sexo, la experiencia docente o el tamaño del centro. 
En el análisis factorial confirmatorio realizado, el constructo resultante consta de cinco factores. El primero de ellos se refiere a la "satisfacción con la formación recibida", el cual consta de siete variables con un alto peso factorial, que incluye la formación permanente en general y aspectos específicos, como la formación en pedagogía y psicología, materias que se imparten, gestión de conflictos del alumnado, función tutorial, trabajo en equipo y normativa educativa.

El segundo factor que se extrae en el análisis factorial indicado es el relativo a la "satisfacción con los horarios laborales y reuniones", que consta de cinco variables con importantes cargas factoriales, y que hacen referencia al horario lectivo, número de horas lectivas y su distribución, así como al número de reuniones y la distribución de su horario.

El tercer factor, integrado por seis variables, se considera en referencia a la "satisfacción con aspectos del centro y las relaciones con directivos y profesorado". En el primer aspecto se considera la satisfacción respecto del ambiente de trabajo, la organización general del centro y la información sobre su funcionamiento. La satisfacción con las relaciones se refiere al profesorado del centro y del departamento, y a los directivos.

Como cuarto factor del constructo, en el análisis factorial confirmatorio, está la "satisfacción con las instalaciones y los recursos materiales y didácticos del centro", con cuatro variables: las instalaciones del centro, los espacios para que el profesor desarrolle su trabajo y los recursos materiales y didácticos de que dispone en el centro.

El último factor hace referencia a la "satisfacción con el reconocimiento del trabajo del profesorado por parte de la administración e inspección y posibilidades de promoción profesional". Las variables que lo integran son el reconocimiento por parte de la administración e inspección educativas, y las posibilidades de promoción profesional.

Se considera que, para incrementar la satisfacción laboral del profesorado de FP, deben mejorarse sus relaciones con "las instituciones educativas", así como el reconocimiento y valoración que tienen éstas de su trabajo. Asimismo, según considera el profesorado consultado, debe prestarse atención a distintos aspectos de su formación. Según Ros-Garrido y García-Rubio (2016), para garantizar la calidad educativa se debe proporcionar una adecuada formación inicial y permanen- 
te al profesorado. Las instituciones y los miembros de la comunidad educativa deben atender las situaciones de acoso, maltrato o agresión sufridas por el profesorado.

Es necesario continuar analizando la satisfacción laboral del profesorado de FP del sistema educativo español, dada la importancia que tiene para su propio desarrollo personal y profesional, y para la calidad de la enseñanza y la evolución que parece sufrir en el tiempo. Este análisis puede incluir otros aspectos, como la autonomía y la autoeficacia del profesor, por su carácter predictivo de la satisfacción laboral (Fradkin-Hayslip, 2021; Skaalvik \& Skaalvik, 2016), y el estrés, la carga de trabajo y el burnout por su relación negativa con la satisfacción laboral (Klassen \& Chiu, 2010; Liu \& Ramsey, 2008).

El análisis factorial confirmatorio realizado tiene una alta consistencia interna y una estructura de cinco factores con índices de bondad de ajuste adecuados. Se entiende que aporta una escala válida y fiable para valorar la satisfacción laboral del profesorado de FP. Como es obvio, el modelo debe ser contrastado en posteriores análisis.

El análisis de la satisfacción laboral del profesorado de FP se espera contribuya a mejorar su situación profesional, con la consiguiente mejora de la calidad de la enseñanza. Administraciones, profesorado y comunidad educativa en general podrán disponer, asimismo, de importante información para implementar los programas que hagan posible dicha mejora

\section{Referencias}

Anaya-Nieto, D. y López Martín, E. (2014). Satisfacción laboral del profesorado en 2012-13 y comparación con los resultados de 2003-04. Un estudio de ámbito nacional. Revista de Educación, (365), 96-121. DOI: https://doi.org/10.4438/1988592X-RE-2014-365-266

Anaya-Nieto, D. y Suárez, J. M. (2006). La satisfacción laboral de los profesores en función de la etapa educativa, del género y de la antigüedad profesional. Revista de Investigación Educativa, 24(2), 541-556. Recuperado de https://revistas.um.es/ rie/article/view/97181

Anaya-Nieto, D. y Suárez, J. M. (2007). Satisfacción laboral de los profesores de educación infantil, primaria y secundaria: Un es- 
tudio de ámbito nacional. Revista de Educación, (344), 217243. Recuperado de www.revistaseducacion.mec.es > re344 re > re344_09

Banerjee, N., Stearns, E., Moller, S. \& Mickelson, R. A. (2017) Teacher job satisfaction and student achievement: The roles of teacher professional community and teacher collaboration in schools. American Journal of Education, 123(2), 1-39. DOI: https://doi.org/10.1086/689932

Baran, M., Maskan, A. \& Baran, M. (2015). Physics, chemistry, and biology teachers' reasons for choosing the profession of teaching and their levels of job satisfaction with respect to certain variables. Journal of Education and Training Studies, 3(3), 101-110. DOI: http://dx.doi.org/10.11114/jets.v3i3.691

Cantón-Mayo, I. y Téllez-Martínez, S. (2016). La satisfacción laboral y profesional de los profesores. Revista Lasallista de Investigación, 13(1), 214-226. DOI: https://doi.org/10.22507/rli. v13nla18

Carrillo, S. (2017). Actitudes hacia la profesión docente y condiciones de bienestar: ¿Una década de cambios y continuidades? Revista Peruana de Investigación Educativa, (9), 5-30. DOI: https://doi.org/10.34236/rpie.v9i9.56

Collie, R. J., Shapka, J. D. \& Perry, N.E. (2012). School climate and social-emotional learning: Predicting teacher stress, job satisfaction and teaching efficacy. Journal of Educational Psychology, 104(4), 1189-1204. DOI: https://doi.org/10.1037/ a0029356

Crossman, A., \& Harris, P. (2006). Job satisfaction of secondary school teachers. Educational Management Administration and Leadership, (34), 29-46. DOI: https://doi. org/10.1177/1741143206059538

Demirtaú, Z. (2010). Teachers' job satisfaction levels. Procedia Social and Behavioral Sciences, (9), 1069-1073. DOI: https://doi. org/10.1016/j.sbspro.2010.12.287

Ferguson, K., Frost, L. \& Hall, D. (2012). Predicting Teacher Anxiety, Depression, and Job Satisfaction. Journal of Teaching and Learning, 8(1), 27-42. DOI: https://doi.org/10.22329/jtl. v8il.2896

Fradkin-Hayslip, A. (2021). Teacher Autonomy, Motivation, and Job Satisfaction: Perceptions of Elementary School Teachers 
According to Self-Determination Theory. Elementary Education Online, 20(2): 198-205. DOI: https://doi.10.17051/ilkonline.2021.02.25

Franco-López, J.A., López-Arellano, H. y Arango-Botero, D. (2020). La satisfacción de ser docente: un estudio de tipo correlacional. Revista Complutense de Educación, 31(1), 55-67. DOI: https://doi.org/10.5209/rced.61775

Gil-Flores, J. (2017). Características personales y de los centros educativos en la explicación de la satisfacción laboral del profesorado. Revista de Psicodidáctica, 22(1). DOI: https://doi. org/10.1016/S1136-1034(17)30039-4

Güell, L. (2014): Estudio de la satisfacción laboral de los maestros. (Tesis doctoral). Universidad Internacional de Cataluña. Recuperado de https://www.redalyc.org/articulo.oa?id=69545978019

Hanushek, E. A., Kain, J. F. \& Rivkin, S. G. (2004). Why public schools lose teachers. Journal of Human Resources, (39), 326-354. DOI: https://doi.org/10.2307/3559017

Hernández-Silva, C., Pavez-Lizarraga, A., González-Donoso, A. y Tecpan-Flores, S. (2017). ¿Se sienten valorados los profesores en Chile? Educación y educadores, 20(3), 434-447. Recuperado de https://intellectum.unisabana.edu.co/ handle/10818/32719?show=full

Hernando-Mora, I. y Sanz-Ponce, R. (2015). La percepción del profesorado de educación secundaria ante la conflictividad escolar. Edetania. Estudios y propuestas socio-educativas, (48), 41-60. Recuperado de https://revistas.ucv.es/index.php/Edetania/ article/view/37

Klassen, R. M. \& Anderson, C. J. K. (2009). How times change: Secondary teachers' job satisfaction and dissatisfaction in 1962 and 2007. British Educational Research Journal, 35(5), 745759. DOI: https://doi.org/10.1080/01411920802688721

Klassen, R. M., \& Chiu, M. M. (2010). Effects on teachers' self-efficacy and job satisfaction: Teacher gender, years of experience, and job stress. Journal of educational Psychology, 102(3), 741-756. DOI: https://doi.org/10.1037/a0019237

Larkin, I. M., Brantley-Dias, L. \& Lokey-Vega, A. (2016). Job satisfaction, organizational commitment, and turnover intention of online teachers in the K-12 setting. Online Learning, 20(3), 26-51. DOI: http://dx.doi.org/10.24059/olj.v20i3.986 
Liu, X. S. \& Ramsey, J. (2008). Teachers' job satisfaction: Analyses of the teacher follow-up survey in the United States for 20002001. Teaching and Teacher Education, 24(5), 1173-1184. DOI: https://doi.org/10.1016/j.tate.2006.11.010

Martín-Ramos, A. (2015). La satisfacción laboral y su relación con el clima organizacional en el ámbito educativo. (Trabajo Final de Grado). Universidad de La Laguna. Recuperado de http:// riull.ull.es/xmlui/handle/915/6344

Martínez-Garrido, C. (2017). La incidencia del liderazgo y el clima escolar en la satisfacción laboral de los docentes en América Latina. Archivos de análisis de políticas de educación, 25(80), 1-22. Recuperado de https://www.redalyc.org/articulo. oa? $\mathrm{id}=275050047065$

MetLife Foundation. (2012). The MetLife Survey of the American Teacher: Teachers, parents, and the economy. MetLife, Inc. Recuperado de http://files.eric.ed.gov/gov/fulltext/ED530021. pdf

Ministerio de Educación y Formación Profesional. Instituto Nacional de Evaluación Educativa. España (2019). Informe PISA 2018. Recuperado de https://www.educacionyfp.gob.es/inee/evaluaciones-internacionales/pisa/pisa-2018.html

Ministerio de Educación y Formación Profesional. Registro estatal de centros docentes no universitarios. Recuperado de https:// www.educacionyfg.gob.es/contenidos/centros-docentes/ buscar-centro-no-universitario.html

Muñoz-Méndez, T., Gómez-Mármol, A. y Sánchez-Alcaraz, B. (2017). Satisfacción laboral en los docentes de educación infantil, primaria y secundaria. Revista Gestión de la Educación, 7(1), 161-177. DOI: https://doi.org/10.46583/edetania_2020.58.635

Naeem-Akhtara, S., Amir-Hashmib, M. \& Hussain-Naqvic, S. I. (2010). A comparative study of job satisfaction in public and private school teachers at secondary level. Procedia Social and Behavioral Sciences, (2), 4222-4228. DOI: https://doi:10.1016/j. sbspro.2010.03.668

National Center for Education Statistics. (1997). Job satisfaction among America's teachers: effects of workplace conditions, background characteristics, and teacher compensation. Washington, D.C., Office of Educational Research and Improvement, 
US Department of Education. Recuperado de https://nces. ed.gov/pubs97471.pdf

Papanastasiou, E. C. \& Zembylas, M. (2005). Job satisfaction variance among public and private kindergarten school teachers in Cyprus. International Journal of Educational Research, 43(3), 147-167. DOI: http://10.0.3.248/j.ijer.2006.06.009

Persevica, A. (2011). The significance of the teacher's job satisfaction in the process of assuring quality education. Problems of education in the 21century, (34), 98-109. Recuperado de http:// journals.indexcopernicus.com/abstract.php?icid=966177

Ramery-Gelpi, E. (2017). La satisfacción laboral del director escolar y los docentes en los centros de secundaria de Orlando, Florida. (Tesis doctoral). Universidad de Jaén. Recuperado de http://hdl. handle.net/10953/892

Raso-Sánchez, F., Sola-Martínez, T. e Hinojo-Lucena, F. J. (2017). Satisfacción del profesorado de la escuela rural de la provincia de Granada (España) respecto a la organización escolar. Bordón, 69(2), 79-96. DOI : https://doi.org/10.13042/Bordon.2017.41372

Renzulli, L. A., Parrott, H. M. \& Beattie, I. R. (2011). Racial mismatch and school type: Teacher satisfaction and retention in charter and traditional public schools. Sociology of Education, 84(1), 23-48. DOI: https://doi.org/10.1177/0038040710392720

Roa-Venegas, J. M. y Fernández-Prados, C. (2020). La motivación de los docentes en la enseñanza secundaria. Revista Reflexión e Investigación Educacional, 2(2), 66-77. Recuperado de http:// revistas.ubiobio.cl/index.php/REINED/article/view/4122

Ros-Garrido, A. y García-Rubio, J. (2016). La calidad en la formación del profesorado del sistema educativo y de los certificados de profesionalidad. Edetania. Estudios y propuestas socioeducativas, (50), 101-119. Recuperado de https://www.mendeley.com/catalogue/41a73818-f397-39f1-b99e-e1990b19c97f/

Sahito, Z. \& Vaisanen, P. (2019). A literature review on teachers' job satisfaction in developing countries: Recommendations and solutions for the enhancement of the job. Review of Education, 8(1), 3-34. DOI: https://doi.org/10.1002/rev3.3159

Santos-Rego, M. A., Godás-Otero, A., Lorenzo-Moledo, M. M. y Gómez-Fraguela, J. A. (2010). Eficacia y satisfacción laboral de 
los profesores no universitarios: Revisión de un instrumento de medida. Revista Española de Pedagogía, 68(245), 151-170. Recuperado de http://hdl.handle.net/10347/16854

Schwartz, G. J. (2017). The relationship between teacher job satisfaction and principal leadership styles. (Dissertation requirements for the Degree Doctor of Education). Carson-Newman University, Jefferson City, Tennessee. USA. Recuperado de https://www.cn.edu/libraries/tiny_mce/tiny:mce/plugins/ filemanager/files/Dissertations/DissertationsFall2017/ Gwendolin_J._Schwartz.pdf

Shobha. M. \& Sarvamangala, D. R. (2020). A study on the job satisfaction of Ashrama school teachers. Research and Reflections on Education, 18(2), 1-9. Recuperado de http://sxcejournal. com/apr-jun-2020/paper4.pdf

Sims, S. (2017). TALIS 2013: Working conditions, teacher job satisfaction and retention (Department for Education Statistical Working Paper). London: Department of Education. Recuperado de https://dera.ioe.ac.uk/30448/1/TALIS_2013_Evidence_on_ Working_Conditions_Teacher_Job_Satisfaction_and_Retention_Nov_2017.pdf

Skaalvik, E. M. \& Skaalvik, S. (2009). Does school context matter? Relations with teacher burnout and job satisfaction. Teaching and Teacher Education, 25(3), 518-524. DOI: https://doi. org/10.1016/j.tate.2008.12.006

Skaalvik, E. M. \& Skaalvik, S. (2016). Teacher stress and teacher selfefficacy as predictors of engagement, emotional exhaustion, and motivation to leave the teaching profession. Creative Education, 7(13), 68-78. DOI: http://doi.org/10.2466/14.02. PRO.114k14w0

Toropova, A., Myrberg, E. \& Johansson, S. (2020). Teacher job satisfaction: the importance of school working conditions and teacher characteristics. Educational Review. DOI: https:// doi.org/10.1080/00131911.2019.1705247

Torres, J. A. (2010). Análisis del grado de satisfacción del profesorado de educación secundaria en el desarrollo de su labor docente. Contextos Educativos, (13), 27-41. DOI: https://doi. org/10.18172/con.624

Veldman, I., Van Tartwijk, J., Brekelmas, M. \& Wubbles, T. (2013). Job Satisfaction and teacher-student relationships across the 
teaching career: Four case studies. Teaching and Teacher Education, (32), 55-65. DOI: https://doi.org/10.1016/j. tate.2013.01.005

Xin, M. \& MacMillan, R. B. (1999) Influences of Workplace Conditions on Teachers' Job Satisfaction. The Journal of Educational Research, 93(1), 39-47. DOI: https://doi. org/10.1080/00220679909597627 